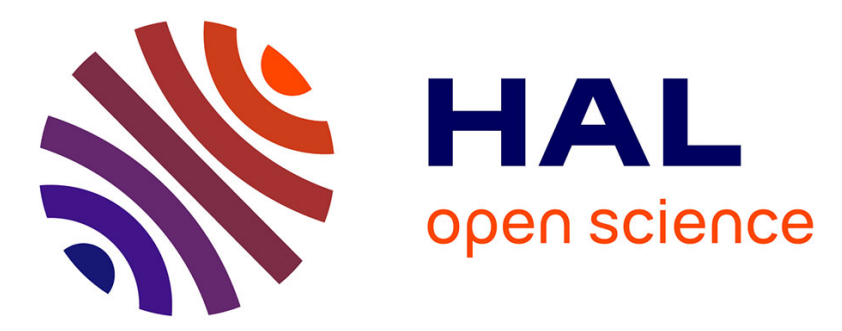

\title{
A novel mouse model of acute graft-versus-host disease based on chemotherapy conditioning and G-CSF mobilized graftsc
}

Yishan Ye, Laure Ricard, Nicolas Stocker, Mohamad Mohty, Beatrice Gaugler, Florent Malard

\section{To cite this version:}

Yishan Ye, Laure Ricard, Nicolas Stocker, Mohamad Mohty, Beatrice Gaugler, et al.. A novel mouse model of acute graft-versus-host disease based on chemotherapy conditioning and G-CSF mobilized graftsc. Bone Marrow Transplantation, 2019, 55 (3), pp.570-577. 10.1038/s41409-019-0700-4 . hal02869581

\section{HAL Id: hal-02869581 \\ https://hal.sorbonne-universite.fr/hal-02869581}

Submitted on 16 Jun 2020

HAL is a multi-disciplinary open access archive for the deposit and dissemination of scientific research documents, whether they are published or not. The documents may come from teaching and research institutions in France or abroad, or from public or private research centers.
L'archive ouverte pluridisciplinaire HAL, est destinée au dépôt et à la diffusion de documents scientifiques de niveau recherche, publiés ou non, émanant des établissements d'enseignement et de recherche français ou étrangers, des laboratoires publics ou privés. 
1 A novel mouse model of acute graft-versus-host disease based on 2 chemotherapy conditioning and G-CSF mobilized graft

3 Running title: A novel GVHD mouse model

4 Yishan Ye, M.D. ${ }^{1,2}$, Laure Ricard, M.D. ${ }^{1}$, Nicolas Stocker, M.D. ${ }^{1}$, Mohamad Mohty M.D., 5 Ph.D. ${ }^{1,3}$, Béatrice Gaugler, Ph.D. ${ }^{1}$, Florent Malard M.D., Ph.D. ${ }^{1,3}$

$6{ }^{1}$ Sorbonne Université, INSERM, Centre de Recherche Saint-Antoine (CRSA), F-75012 Paris, 7 France

$8{ }^{2}$ Bone Marrow Transplantation Center, The First Affiliated Hospital, School of Medicine, 9 Zhejiang University, Hangzhou, 310003, China

$10{ }^{3}$ AP-HP, Hôpital Saint-Antoine, Service d'Hématologie Clinique et Thérapie Cellulaire, 11 F-75012, Paris, France

13 Correspondence and reprint requests:

14 Florent Malard, M.D., Ph.D.; Service d'Hématologie Clinique et de Thérapie Cellulaire, 15 Hôpital Saint Antoine, APHP, 184 rue du Faubourg Saint-Antoine, 75012, Paris, France. 16 Phone : +33 149282629 ; Fax : +33 149283375 Email : florent.malard@inserm.fr 


\section{Abstract}

19 Acute graft-versus-host disease (aGVHD) is an important complication of allogeneic

20 hematopoietic cell transplantation (HCT). The majority of aGVHD mouse models are based

21 on radiation conditioning and bone marrow as graft, despite that most allo-HCTs performed

22 now in clinic are based on chemotherapy conditioning and G-CSF mobilized graft. Aiming

23 for a higher translational value, we constructed an MHC major mismatched [C57BL/6 (H-2

$24 \mathrm{~Kb}$ ) to $\mathrm{BALB} / \mathrm{c}(\mathrm{H}-2 \mathrm{Kd})$ ] aGVHD mouse model based on busulfan/cyclophosphomide

25 (BU-CY) conditioning and human G-CSF mobilized splenocytes as graft. Allogeneic

26 transplanted mice showed quick and profound donor engraftment. Moreover, there were

27 quick onset (day +7 ) of typical clinical and histopathological signs of aGVHD, which

28 gradually developed to extensive aGVHD. In addition, CD8+ T cells were the main aGVHD

29 contributing $\mathrm{T}$ cell subtype. No toxicity or GVHD signs were observed in the syngeneic

30 setting. This clinical relevant model offers a promising platform for future studies on

31 aGVHD. 
Acute graft-versus-host disease (aGVHD) is an important inflammatory complication of

40 allogeneic hematopoietic cell transplantation (HCT) [1, 2]. Poor translational value remains

41 the limitation for many aGVHD mouse models. The majority of aGVHD mouse models are based on total body irradiation (TBI) as conditioning and bone marrow (BM) as graft, despite that most allo-HCTs performed now in clinic are based on chemotherapy conditioning and G-CSF mobilized grafts [3]. van Leeuwen et al constructed the classical MHC major mismatched $\mathrm{C} 57 \mathrm{BL} / 6(\mathrm{H}-2 \mathrm{~Kb})$ to BALB/c $(\mathrm{H}-2 \mathrm{Kd})$ model based on TBI and bone marrow graft [4]. Subsequently, Sadeghi et al. constructed the first major histocompatibility complex

47 (MHC) major mismatched [C57BL/6 (H-2Kb) to BALB/c $(\mathrm{H}-2 \mathrm{Kd})]$ model based on busulfan/cyclophosphamide (BU-CY) conditioning [5]. Afterwards, some other chemo-based aGVHD mouse models were also developed to investigate the different contexts of 50 haploidentical [6] and miHA mismatched [7] allo-HCTs. Moreover, aGVHD models based on 51 TBI and G-CSF mobilized grafts has been described [8]. However, none of them have 52 combined these two clinically relevant factors in one model.

53 Aiming for a higher translational value, we developed a MHC major mismatched aGVHD 54 model using $\mathrm{C} 57 \mathrm{BL} / 6(\mathrm{H}-2 \mathrm{~Kb}$, female) as donor and BALB/c (H-2Kd, female) as recipient. 55 This model manifested typical clinical and histological signs of aGVHD, and may play as a 56 promising clinical-relevant model for future studies. 
Materials and Methods

60

\section{Mice}

Female C57BL/6j mice $(\mathrm{H} 2 \mathrm{~kb})$ and female $\mathrm{BALB} / \mathrm{c}(\mathrm{H} 2 \mathrm{kd})$ mice were purchased from Janvier (Genest-St-Isle, France). All mice were used at 11-13 weeks of age. Mice were maintained under pathogen free controlled conditions and $12 \mathrm{hr}$ light/darkness. Animals had access to food and water ad libitum. All protocols were performed according to approval of the "Service Vétérinaires de la Santé et de la Protection Animal" delivered by the Ministry of Agriculture of France.

\section{HCT procedures}

BU (Pierre Fabre Médicament, Idron, UK) and CY (Baxter, Saint-Quentin-en-Yvelines, France) were used for conditioning. BU $(4 \mathrm{mg} / \mathrm{ml})$ was diluted with PBS, and CY $(20 \mathrm{mg} / \mathrm{ml})$ was injected without dilution. Female BALB/c mice received IP doses of BU (20 or 25 $\mathrm{mg} / \mathrm{kg} / \mathrm{d}$ ) for 4 days (day -7 to day -4$)$, followed by CY $(100 \mathrm{mg} / \mathrm{kg} / \mathrm{d})$ for 2 days (day -3 to day -2). After 1 day rest, the recipient mice were injected IV with $10 \times 10^{6}$ splenic cells from female C57BL/6 mice by tail vein injection. PBS was administrated as a vehicle control. For graft preparation, donors were previously treated by subcutaneous injection of $10 \mu \mathrm{g}$ per animal of recombinant human G-CSF (Hospira, Hurley, UK) once daily from day -5 to day -1 .

On the day of transplant, the donors were sacrificed by cervical dislocation. Donor spleens were disrupted in RPMI-1640 culture medium (Thermo Fisher Scientific, Villebon-sur-Yvette, France) and erythrocytes were then lysed with RBC Lysis Buffer (Multi-species) (Thermo Fisher Scientific, Villebon-sur-Yvette, France), washed twice with RPMI-1640 before passing 
through a $70 \mathrm{~mm}$ strainer. Cells were then resuspended in PBS for injection. Cell viability

$81(>90 \%)$ was confirmed with trypan blue. Each experiment included 5 to 6 mice from each

82 group. The Chemo group $(\mathrm{BALB} / \mathrm{c})$ received chemotherapy alone without infusion of splenocytes, the Allo group (BALB/c) received chemotherapy followed by infusion of allogeneic splenocytes (C57BL/6), and the Syn group (BALB/c) received chemotherapy

85 followed by infusion of syngeneic splenocytes (BALB/c). The mice were randomly assigned to Chemo, Allo or Syn groups in each experiment. No mice were excluded from analysis. No statistical methods were used to predetermine sample size. The investigators were not blinded to allocation during experiments and outcome assessment.

\section{Clinical and histological assessment of GVHD}

Mice were monitored for survival and individually scored every two days for five clinical parameters (posture, activity, fur, skin and weight loss) from conditioning initiation until the appropriate sampling day on a scale from 0 to 2 as previously described [9]. Mice survival was the primary endpoint for GVHD evaluation. Clinical GVHD score was assessed by summation of these parameters. Animals with severe aGVHD (scores $>6$ ) were sacrificed according to ethical guidelines. For histological analysis, the skin and colon were removed immediately after sacrifice on day $+7,+14$ and +21 after transplantation, and then fixed in $4 \%$

97 formalin for $24 \mathrm{~h}$, transferred to $70 \%$ ethanol, dehydrated and embedded in paraffin. Paraffin sections were then stained with hematoxylin and eosin. The histological grade of skin and intestine aGVHD was assessed according to the grading system previously described [10].

100 Scores were determined in a blinded fashion by two pathologists. 
101

102

103

104

105

106

107

108

109

110

111

112

113

114

\section{FACS analysis}

On day $+7,+14,+21$ and +28 after transplantation, one random recipient from each group was sacrificed, and splenocytes and BM cells were collected. BM and spleen cellularity were determined by trypan blue staining and microscopic count. Afterwards erythrocytes were lysed with RBC Lysis Buffer and cells were then passed through a $40 \mu \mathrm{m}$ cell strainer, washed twice and stained for 20 minutes at $4{ }^{\circ} \mathrm{C}$ in $\mathrm{PBS} / 0.5 \mathrm{mM}$ EDTA/0.5\% BSA with the following mouse mAbs: FITC-CD8a (SONY, Weybridge, UK), PE-CD4 (SONY), PerCPCy5.5-CD11c (SONY), PC7-H2Db (Biolegend, Ozyme, Saint-Quentin-Fallavier, France), A647-H2Dd (Biolegend), APCCY7-CD3 (SONY) and PACIFIC BLUE-CD45 (Biolegend). Analyses were performed with CytoFLEX Flow Cytometer (Beckman Coulter) and Kaluza Flow Cytometry Analysis Software version 1.5a (Beckman Coulter).

\section{Statistical analysis}

Survival data were analyzed using the Kaplan-Meier method and Mantel-Cox log-rank test. For all other data, the two-sided unpaired t-test was used. Normality tests and the F test confirmed Gaussian distribution and equality of variance between different groups. Values were presented as mean \pm standard error of the mean $(\mathrm{SEM})$. A value of $\mathrm{P}<0.05$ was considered statistically significant in all experiments. Data was computed using GraphPad Prism 5.0 (GraphPad Software).

\section{Results}

For dose optimization purpose, two different doses of BU were evaluated (80 and $100 \mathrm{mg} / \mathrm{kg}$ 
122

123

124

125

126

127

128

129

130

131

132

133

134

135

136

137

138

139

140

141

142

143

total dose) in combination with a fixed dose of CY $(100 \mathrm{mg} / \mathrm{Kg})$. While dose of BU has no impact on mice survival in the syngeneic setting (groups 3 and 4, Figure 1a), higher dose of BU (100 mg/kg) was associated with very early death, within 10 days, both in the Chemo and the Allo group (groups 6 and 2, figure 1a). On the contrary, with lower dose of BU (80 mg/kg) early deaths were slightly delayed in the Allo group comparing to the Chemo group (groups 1 and 5, figure 1a). Overall GVHD onset and kinetic with the lower dose of BU $(80 \mathrm{mg} / \mathrm{kg})$ was more adapted and BU $80 \mathrm{mg} / \mathrm{kg}$ in combination with CY $200 \mathrm{mg} / \mathrm{kg}$ which was retained has the optimal conditioning regimen for further experiments.

The protocol for transplantation and G-CSF mobilization is shown in Figure $1 b$. Appropriately conditioned and syngeneic transplanted mice (Syn group) survived over +60 days of transplant, with no signs of aGVHD. Mice who received conditioning but no transplantation (Chemo group) started to die from day +2 and $64.7 \%$ (11 out of 17 mice) died within 15 days. Appropriately conditioned and allogeneic transplanted mice (Allo group) started to die from day +4 , with $76.5 \%$ (13 out of 17 mice) died within 20 days, and all died within 40 days (median survival=12 days) (Figure $1 \mathrm{c}$ ). In all groups, a weight loss was seen between day -7 and day +5 . In the Allo group, weight reached the nadir on day +7 and remained at a low level, until the second phase of weight loss on day +30 (Figure 1d). Meanwhile, features of aGVHD including hunched posture (Figure 2a), hair loss (Figures $2 b, c)$ and ruffled fur (Figure 2c) were observed in the Allo group. Moreover, almost all mice in the Allo group manifested signs of colitis from day +5 until the end of the experiment. Finally, clinical GVHD scores were assessed as previously described [8], and represented the severity of aGVHD (Figure 2d). 
144 With fluorescence activated cell sorting (FACS) analysis, we observed a stable donor

chimerism of over $95 \%$ in $\mathrm{BM}$, and an increasing donor cell chimerism in the spleen, from $68 \%$ from day +7 to full chimerism on day +14 in the Allo group (Figure 3a). T cell engraftment was faster than that of granulocytes in the spleen (Figure 3b). BM and spleen cellularity recovered rapidly in the Syn group. However in the Allo group, the recovery of BM and spleen was slower and at day +28 reached $65 \%$ and $71 \%$, respectively when compared with the Syn group (Figure 3c). As previously published [5], percentages of CD3 $+\mathrm{T}$ cells were significantly increased in the BM and spleen early after transplant due to chemotherapy conditioning (day +7$)$, and decreased gradually until day +28 . However, the percentages of CD3 $+\mathrm{T}$ cells were always higher in the Allo group as compared with the Syn group (Figure $4 a, c)$, and the frequencies of CD8+ T cells, but not CD4+ cells, were significantly higher in the Allo group as compared with the Syn group, in both BM and spleen (Figure $4 b, d$ ). The ratio of $\mathrm{CD} 4 / \mathrm{CD} 8$ was reversed consistently in the Allo group from day +7 until day +28 in both BM and spleen (Figure 4e).

At day +7 the Allo group exhibited significant histopathologic skin changes. Frequent apoptotic figures were observed within the epidermis of the dorsal skin, as well as subepidermal vesical formation, lymphocytic infiltration and slight lifting of the epidermal layer. The Chemo group also manifested epidermis vacuolization probably due to conditioning toxicity, but no immune cell infiltration was observed. The Syn group maintained an essentially intact epidermal and dermal layer (Figure 5a). At day +21 , the interfollicular epidermal hyperplasia was extensive and dyskeratotic squamous cells had spread throughout the epidermis. A high density of immune cells had infiltrated the epidermis. 
At day +7 , the Allo group exhibited colon histopathologic changes including crypt cell apoptosis and immune cell infiltration. Crypt damage was also seen in the Chemo group probably due to conditioning toxicity. The Syn group, however, exhibited an essentially normal structure (Figure 5a). At day +21 , the crypt structure in the Allo group was severely disturbed with extensive crypt destruction and dense lamina propria lymphocytic infiltration. The Chemo and Syn group however, manifested normal intestinal structure (Figure 5b).

The histopathological scores were assessed as previously described [10]. The scores were higher in the Allo group than in the Syn group for both skin and colon at day +7 (Figure 6a). By day +21 , the Allo group showed significantly increased histopathological scores as compared with the Syn group for skin (Allo: $2.5 \pm 0.3$ vs Syn: $0.0 \pm 0.0 ; \mathrm{P}<0.0001$ ) and colon (Allo: $8.7 \pm 0.2$ vs Syn: $3.2 \pm 0.6 ; \mathrm{P}<0.0001$ ). Meanwhile, the scores of the Allo group was also significantly higher than those of the Chemo group for both skin (Allo vs Chemo: $0.0 \pm$ $0.0 ; \mathrm{P}=0.0016$ ) and colon (Allo vs Chemo: $5.7 \pm 0.3 ; \mathrm{P}<0.0001$ ).

\section{Discussion}

So-far the great majority of aGVHD mouse models have been based on TBI as conditioning and BM as graft [3]. However, the use of lethal TBI without chemotherapy in mouse models is in contrast to the standard procedure in clinical allo-HCT, and profound differences between these two types of conditioning regimens may lead to a distinct GVHD phenotype. Moreover, immune cells derived from different sources (BM or G-CSF mobilized graft) 
187

might have different trafficking capacities and composition, and therefore exert distinct influence on the GVHD phenotype [11].

Compare to the classical MHC major mismatched $\mathrm{C} 57 \mathrm{BL} / 6(\mathrm{H}-2 \mathrm{~Kb})$ to BALB/c $(\mathrm{H}-2 \mathrm{Kd})$ model based on TBI and bone marrow graft [4], median survival in the Allo group was shorter in our model, being 12 days, versus 18 days in the classical model, suggesting that our model may represent a more typical acute phase of GVHD.

Sadeghi et al. established conditioning with BU-CY in a MHC mismatched model using bone marrow graft [5]. After dose optimization, we use the exact same dose of chemotherapy, BU-CY $(80 \mathrm{mg} / \mathrm{kg}-200 \mathrm{mg} / \mathrm{kg})$, in our model, with a lethality of $65 \%$ in the Chemo group comparable to the study by Sadeghi et al [5]. However, despite BU-CY was not fully myeloablative, chimerism analysis demonstrate that all mice achieved full donor engraftment with either bone marrow[5] or G-CSF mobilized grafts after allo-HCT.

Weight loss was the most well documented manifestation in the GVHD mouse model [3]. In our study, the Allo group manifested weight loss between day -7 and day +7 and remained low afterwards. This weight change indicated a quick onset of GVHD. This early weight loss in the Allo group was comparable to that observed in the model using BU-CY conditioning and bone marrow graft [5]. However, when the follow-up duration was prolonged, we observed a second phase of weight loss which led to death of all mice by day +40 , indicating that aGVHD was lethal and progressive in this model.

Concerning immune reconstitution, $\mathrm{T}$ cell engraftment in the current model seems to be quicker in the GVHD target organs when comparing to the model using bone marrow as graft. 
208 At day +14 , we observed $95.5 \%$ of donor $\mathrm{T}$ cell chimerism in the recipient spleen, while at the same time point donor chimerism was around $70 \%$ in mice receiving bone marrow as

210 reported [5]. This observation is consistent the clinical report that recipients of

211 G-CSF-mobilized grafts from unrelated donors have faster immune reconstitution than BM 212 transplant recipients [14]. Moreover, the T cell engraftment was slower than granulocytes in 213 the spleen, which is consistent with the kinetic of immune reconstitution after Allo-HCT in 214 humans [15].

215 A reversed $\mathrm{CD} 4 / \mathrm{CD} 8$ ratio was observed in the Allo group BM and spleen throughout the 216 process of aGVHD, indicating a disease process largely driven by CD8+ T cells. In Sadeghi 217 et al's model CD8 $+\mathrm{T}$ cells also played the major pathogenetic role [5]. In the classic $218 \mathrm{C} 57 \mathrm{BL} / 6$ to $\mathrm{BALB} / \mathrm{c}$ with TBI and bone marrow graft model a two-phase pathogenesis of 219 GVHD was observed and studies using transgenic mice that have a mutant MHC I or MHC II revealed that CD8+ $\mathrm{T}$ cells were the early and leading cause of GVHD mortality (acute 221 phase), while the latter phase was probably mediated by alloreactive CD4+ T cells $[11,12]$. 222 Overall, our findings are in accordance with previously published data, with early CD8+ T 223 cell drived aGVHD mortality. Further study would be useful to decipher the exact role of T 224 cell subset and in particular to rule out a potential role of CD4+ T cells in latter aGVHD 225 death.

226 Typical clinical and histopathological signs of aGVHD were observed in our model. 227 Extensive intestinal aGVHD was largely responsible for the weight loss and final death. 228 Moreover, evident pathological alterations including infiltration of inflammatory cells into 229 the skin and large intestine and the presence of high numbers of apoptotic/necrotic cells in all 
tested organs were similar to those observed in irradiation-based aGVHD mouse models and human aGVHD $[1,2]$.

232 We cannot rule out that chemotherapy toxicity contributed to early death in the Allo group. In

233 fact, lesions induced by conditioning regimen, in particular gut lesions, directly contribute to 234 aGVHD initiation, that once initiated will aggravate the lesion, leading to mice death. 235 However, bone marrow and spleen engraftment with CD8+ $\mathrm{T}$ cell infiltration in the Allo 236 group, along with specific impairment in those mice (fur loss, colitis) and increased 237 histopathology score at day 21, confirm that aGVHD was the leading cause of death in those 238 mice.

Overall, this clinically relevant model showed good donor engraftment and typical clinical and histopathological signs of aGVHD, which may be advantageous in reflecting the clinical situation of HCT and aGVHD. There are limitations to this model. Firstly, the one-week long conditioning is a more complicated practice as compared with the 'single shot' TBI. In addition, this is a MHC major mismatched model, while in clinic, minor MHC mismatched and haploidentical HCTs are more routinely practiced. Therefore, haploidentical or minor MHC mismatched models based on chemotherapy conditioning and G-CSF mobilized grafts 246 are expected in the future.

\section{Acknowledgments}

249 The authors acknowledge the Association for Training, Education and Research in 250 Hematology, Immunology and Transplantation for the generous and continuous support to the 
research work. Y.Y thanks the China Scholarship Council for financial support (CSC No. 201606320257).

\section{Conflict of Interest}

255 The authors state no conflict of interest.

\section{References}

2601 Ferrara JL, Levine JE, Reddy P, Holler E. Graft-versus-host disease. Lancet 2009; 373:

$261 \quad 1550-1561$.

2622 Holtan SG, Marcelo P, Weisdorf DJ. Acute graft-versus-host disease: A bench-to-bedside 263 update. Blood 2014; 124: $363-373$.

2643 Schroeder MA, DiPersio JF. Mouse models of graft-versus-host disease: advances and 265 limitations. Dis Model Mech 2011; 4: 318 - 333.

2664 van Leeuwen L, Guiffre A, Atkinson K, Rainer SP, Sewell WA. A two-phase pathogenesis 267 of graft-versus-host disease in mice. Bone Marrow Transplant 2002; 29: 151 - 158. 
5 Sadeghi B, Aghdami N, Hassan Z, Forouzanfar M, Rozell B, Abedi-Valugerdi M et al. GVHD after chemotherapy conditioning in allogeneic transplanted mice. Bone Marrow Transplant 2008; 42: $807-818$.

6 Li N, Hu W, Li X, Yang W, Chen P, Chen Y. [Establishment of a graft-versus-host disease mouse model for haplo-identical bone marrow transplantation with busulfan and fludarabine conditioning regimen]. Zhonghua Xue Ye Xue Za Zhi 2015; 36: 238-240b.

7 Riesner K, Kalupa M, Shi Y, Elezkurtaj S, Penack O. A preclinical acute GVHD mouse model based on chemotherapy conditioning and MHC-matched transplantation. Bone Marrow Transplant 2016; 51: $410-417$.

8 Arbez J, Saas P, Lamarthée B, Malard F, Couturier M, Mohty M et al. Impact of donor hematopoietic cells mobilized with G-CSF and plerixafor on murine acute graft-versus-host-disease. Cytotherapy 2015; 17: 948 - 955.

9 Cooke K, Kobzik L, Martin T, Brewer J, Delmonte JJ, Crawford J et al. An experimental model of idiopathic pneumonia syndrome after bone marrow transplantation: I. The roles of minor $\mathrm{H}$ antigens and endotoxin. Blood 1996; 88: 3230 - 3239.

10 Couturier M, Lamarthee B, Arbez J, Renauld JC, Bossard C, Malard F et al. IL-22 deficiency in donor $\mathrm{T}$ cells attenuates murine acute graft-versus-host disease mortality while sparing the graft-versus-leukemia effect. Leukemia 2013; 27: 1527 - 1537.

11 Stolfi JL, Pai CCS, Murphy WJ. Preclinical modeling of hematopoietic stem cell transplantation - Advantages and limitations. FEBS J 2016; 283: 1595 - 1606. 
288

289

290

291

292

293

294

295

296

297

298

299

300

301

302

303

304

305

306

307
12 Maeda Y, Levy RB, Reddy P, Liu C, Clouthier SG, Teshima T et al. Both perforin and Fas ligand are required for the regulation of alloreactive CD8 $+\mathrm{T}$ cells during acute graft-versus-host disease. Blood 2005; 105: 2023-2027.

13 Anderson BE, Mcniff JM, Jain D, Blazar BR, Shlomchik WD, Shlomchik MJ. Distinct roles for donor- and host-derived antigen-presenting cells and costimulatory molecules in murine chronic graft-versus-host disease : requirements depend on target organ. Blood 2005; 105: $2227-2234$.

14 Waller EK, Logan BR, Fei M, Lee SJ, Confer D, Howard A et al. Kinetics of immune cell reconstitution predict survival in allogeneic bone marrow and G-CSF - mobilized stem cell transplantation. Blood Adv 2019; 3: 2250 - 2263.

15 Ogonek J, Kralj JM, Ghimire S, Varanasi P, Holler E, Greinix H et al. Immune Reconstitution after Allogeneic Hematopoietic Stem Cell Transplantation. Front Immunol $2016 ;: 507$.

16 Ferrara JL, Levine JE, Reddy P, Holler E. Graft-versus-host disease. Lancet. 2009; $373: 1550-1561$. 


\section{Figure Legends}

312

313

\section{Figure 1. HCT protocol and survival}

(a) Survival curve of mice underwent different treatments. Group 1: Female BALB/c conditioned with BU - CY (80 - $200 \mathrm{mg} / \mathrm{kg})$ and transplanted with female C57BL/6; Group 2: Female BALB/c conditioned with BU - CY $(100-200 \mathrm{mg} / \mathrm{kg})$ and transplanted with female C57BL/6; Group 3: Female BALB/c conditioned with BU - CY $(80-200 \mathrm{mg} / \mathrm{kg})$ and transplanted with female BALB/c; Group 4: Female BALB/c conditioned with BU - CY $(100-200 \mathrm{mg} / \mathrm{kg})$ and transplanted with female BALB/c; Group 5: Female BALB/c conditioned with BU - CY $(80-200 \mathrm{mg} / \mathrm{kg})$ and not transplanted; Group 6: Female BALB/c conditioned with BU - CY $(100-200 \mathrm{mg} / \mathrm{kg})$ and not transplanted. All transplanted mice received $10 \times 10^{6}$ splenic cells from donor origin. (b) Female BALB/c received IP doses of BU-CY $(80 \mathrm{mg} / \mathrm{kg}-200 \mathrm{mg} / \mathrm{kg})$ per mouse beginning at day -7 before HCT. For transplantation, $10 \times 10^{6}$ splenic cells from G-CSF mobilized C57BL/6 mice were given by tail vein injection on day 0. (c) and (d) show data from 17 mice per group from 3 independent experiments. (c) Survival curve, ${ }^{* * *} \mathrm{p}<0.001$ by Mantel-Cox log-rank test. (d) Mean \pm SEM of mouse weight in aGVHD phase, $* * * \mathrm{p}<0.001$ by t-test.

\section{Figure 2. GVHD Clinical manifestations}

The Allo group exhibited features of aGVHD including hunched posture (a), hair loss (b, c), and ruffled fur (c). (d) Mean \pm SEM of clinical GVHD score from 3 independent experiments, $* * * p<0.001$ by t-test. Clinical GVHD score was assessed by summation of five parameters (posture, activity, fur, skin and weight loss). 
332

333

\section{Figure 3. Donor engraftment}

(a) Donor chimerism (\%) in BM and spleen in Allo group. (b) T cell and granulocyte (GN) engraftment in the spleen in Allo group. (c) BM and spleen cellularity during GVHD, as determined by trypan blue staining and microscopic count. Each time point represents Mean \pm SEM of data from at least 3 independent mice.

\section{Figure 4. Lymphoid subpopulations}

(a) $\mathrm{T}$ cell (\%) in BM. (b) CD8+ and CD4+ T cell (\%) in BM. (c) $\mathrm{T}$ cell (\%) in spleen. (d) CD8+ and CD4+ $\mathrm{T}$ cell (\%) in spleen. (e) CD4/CD8 ratio in BM and spleen. Each time point represents Mean \pm SEM of data from at least 3 independent mice. $* * p<0.01$ and $* * * p<0.001$ by t-test.

\section{Figure 5. GVHD pathophysiology}

At day +7 (a) and day +14 (b) after HCT, mice in the Syn and Allo group were sacrificed for histological examination. Representative H\&E-stained sections of dorsal skin and colon from each group are shown. Original magnification was $\times 200$.

\section{Figure 6. GVHD histopathological score}

Skin and intestinal aGVHD histopathological scores at day +7 (a) and day +21 (b) after HCT. Scores of skin and intestinal GVHD were significantly higher in the Allo group than in the Syn and the Chemo group at $\mathrm{d}+21$ after HCT, respectively. Data are shown as Mean \pm SEM, $* * * \mathrm{p}<0.001$ by t-test. 
Figure 1

a

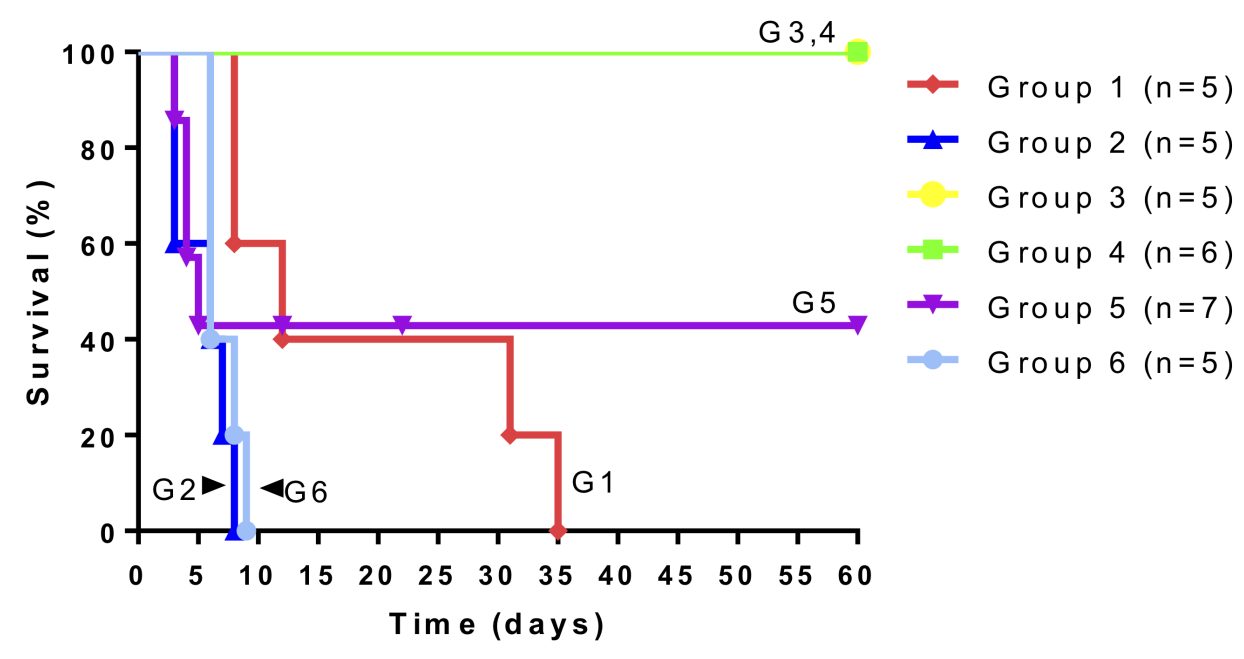

b

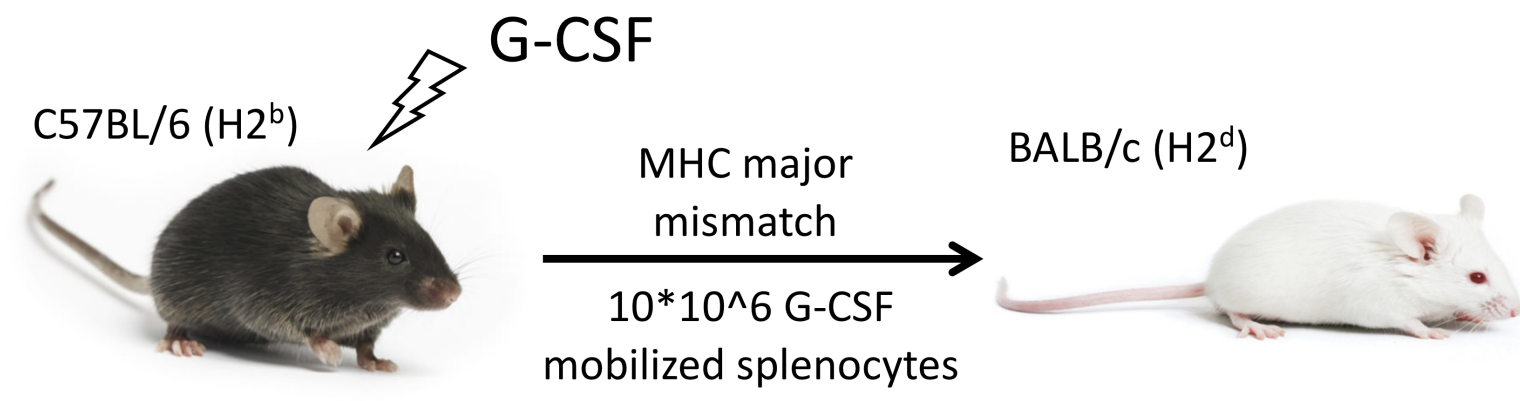

Chemotherapy BALB/C

day -7 day -6 day -5 day -4 day -3 day -2 day -1 day 0

Conditioning regimen

\section{$\mathrm{BU} 80 \mathrm{mg} / \mathrm{kg}$}

Donor G-CSF mobilization

\section{CY $200 \mathrm{mg} / \mathrm{kg}$}

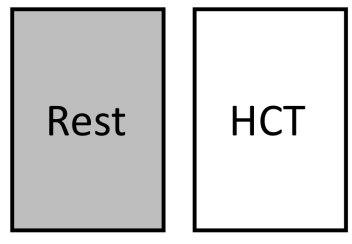

G-CSF $10 \mu \mathrm{g} /$ mouse/day

C

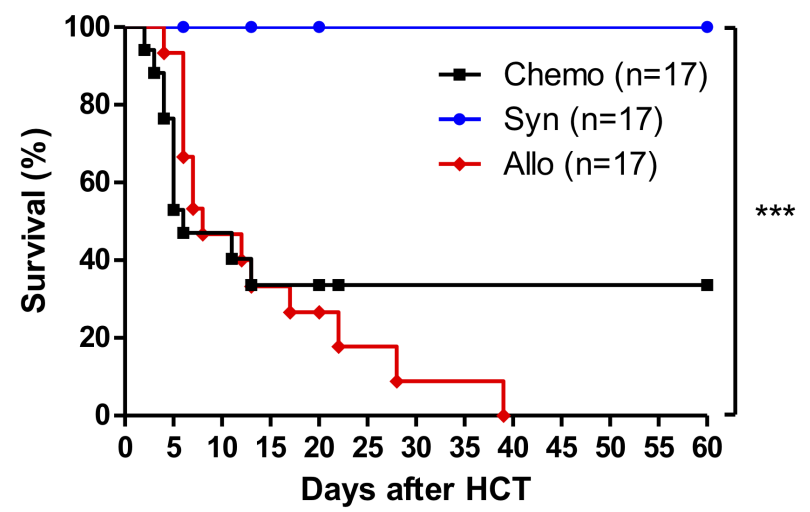

d

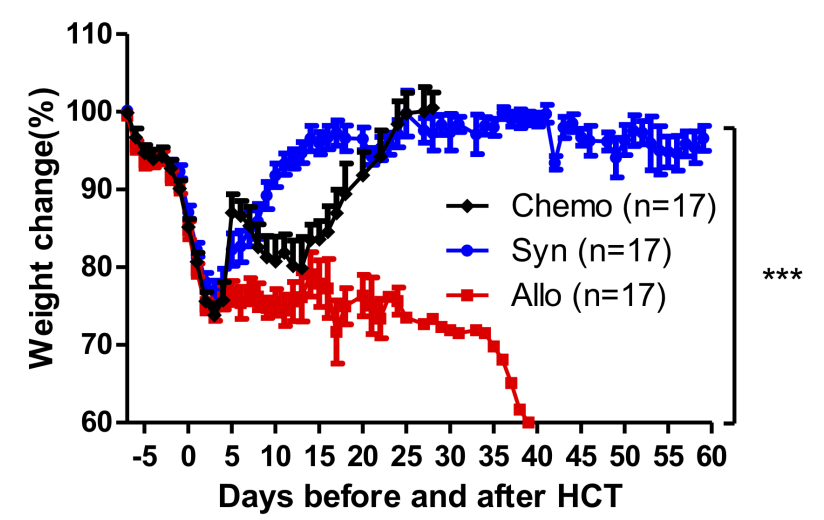


Figure 2

a

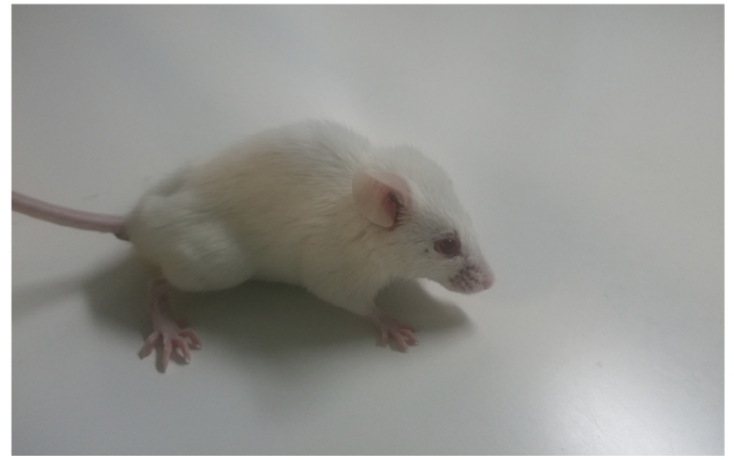

C

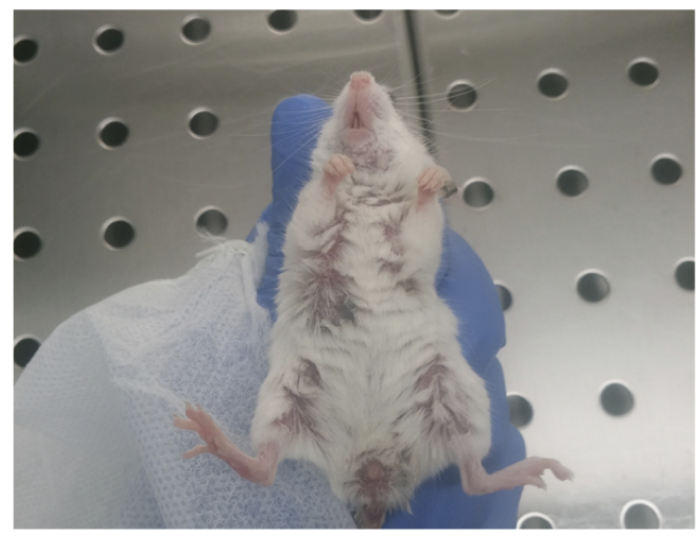

b

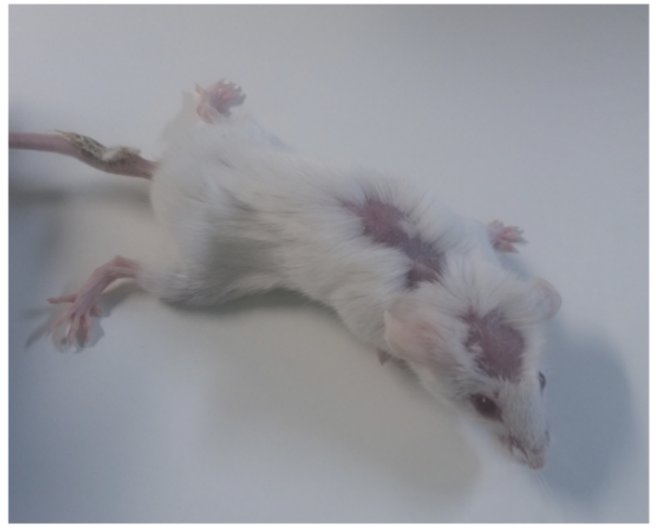

d

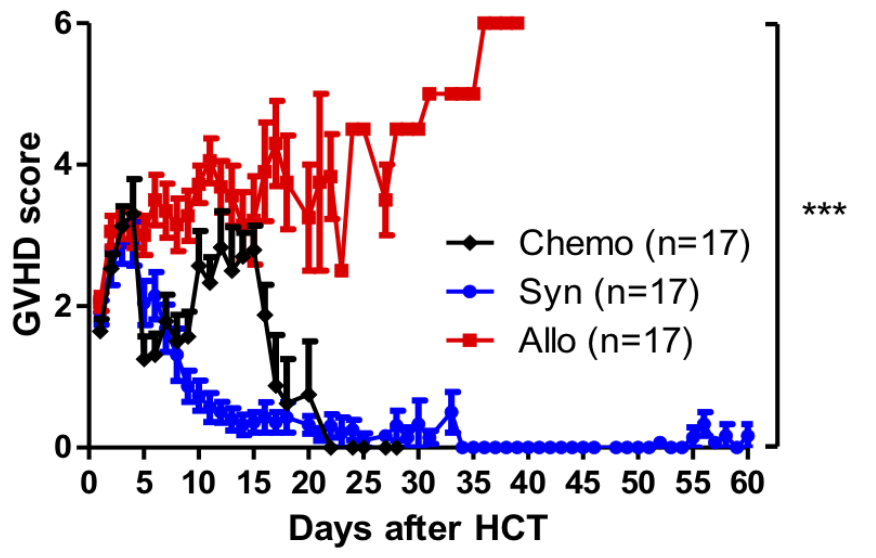


Figure 3

a

Allogeneic donor BM and spleen chimerism

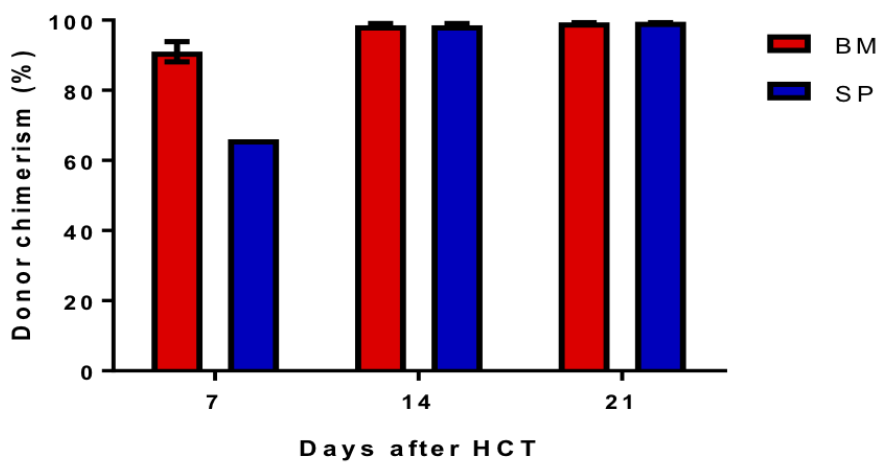

C

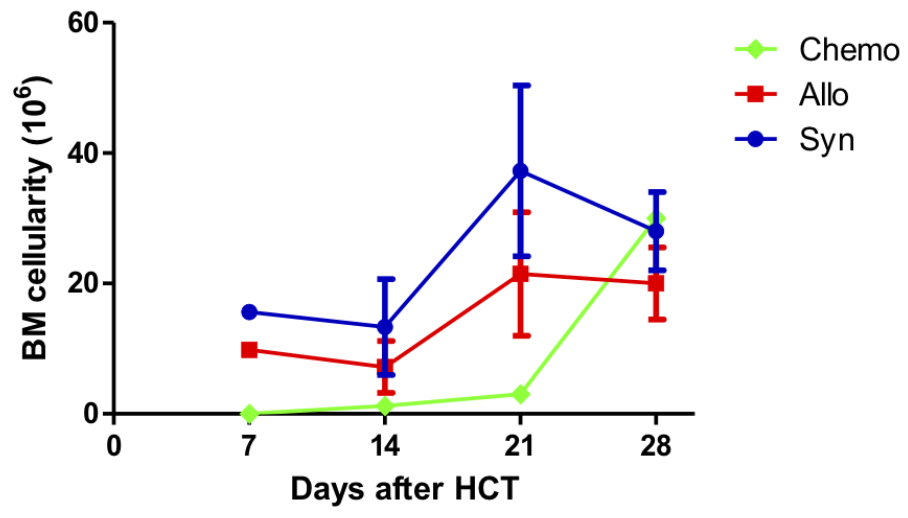

b

Allogeneic donor T/GN chimerism in spleen
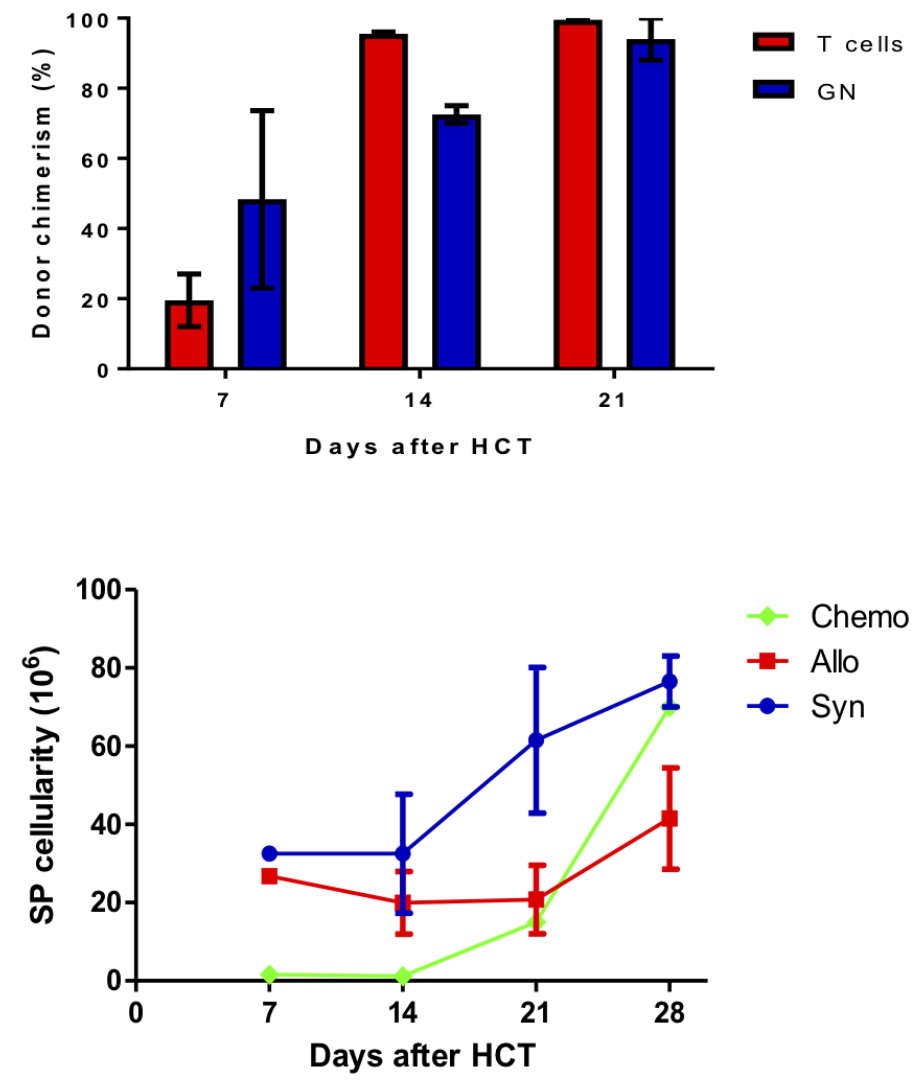


\section{Figure 4}

a

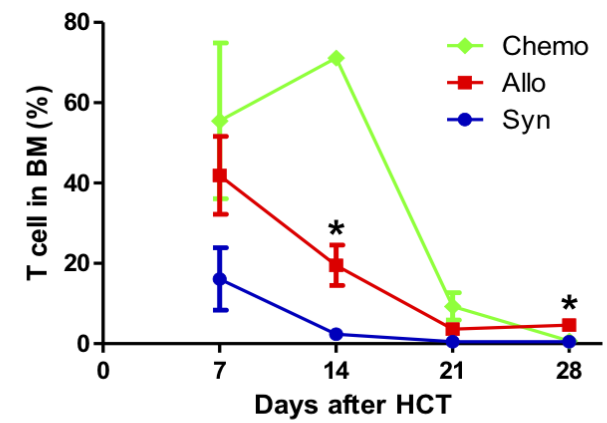

C

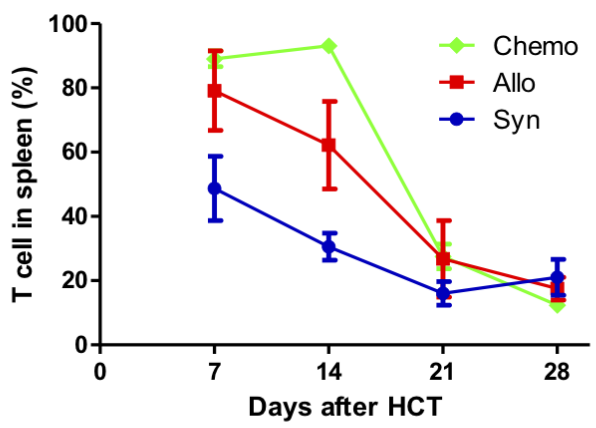

b

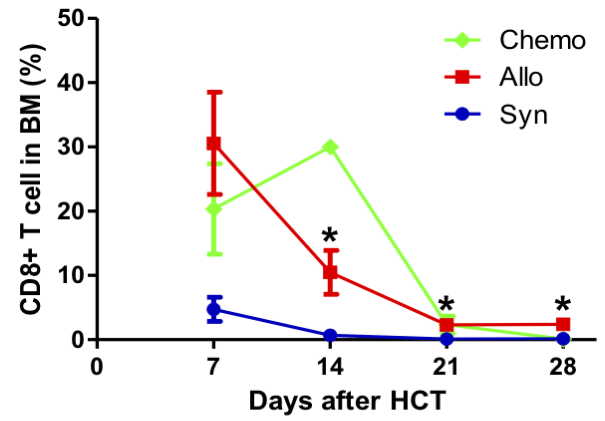

d

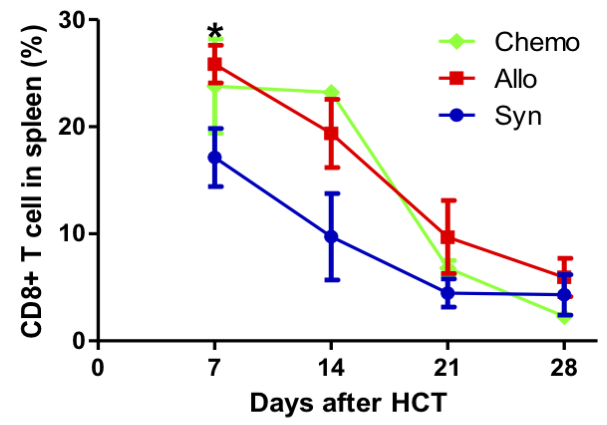

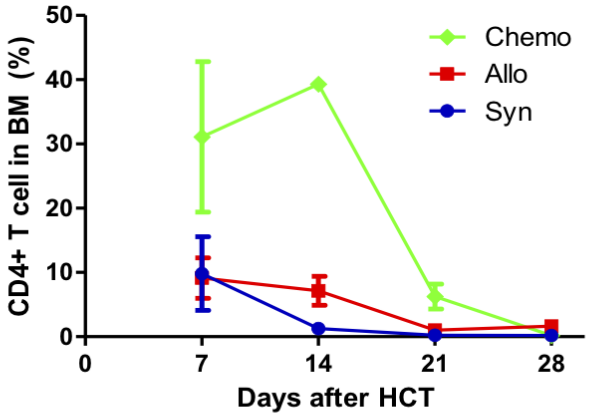

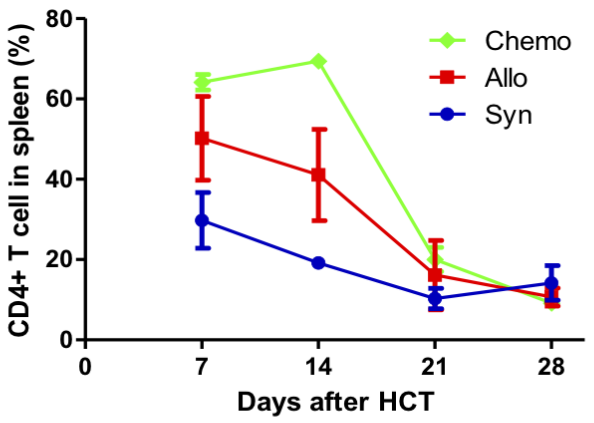

e
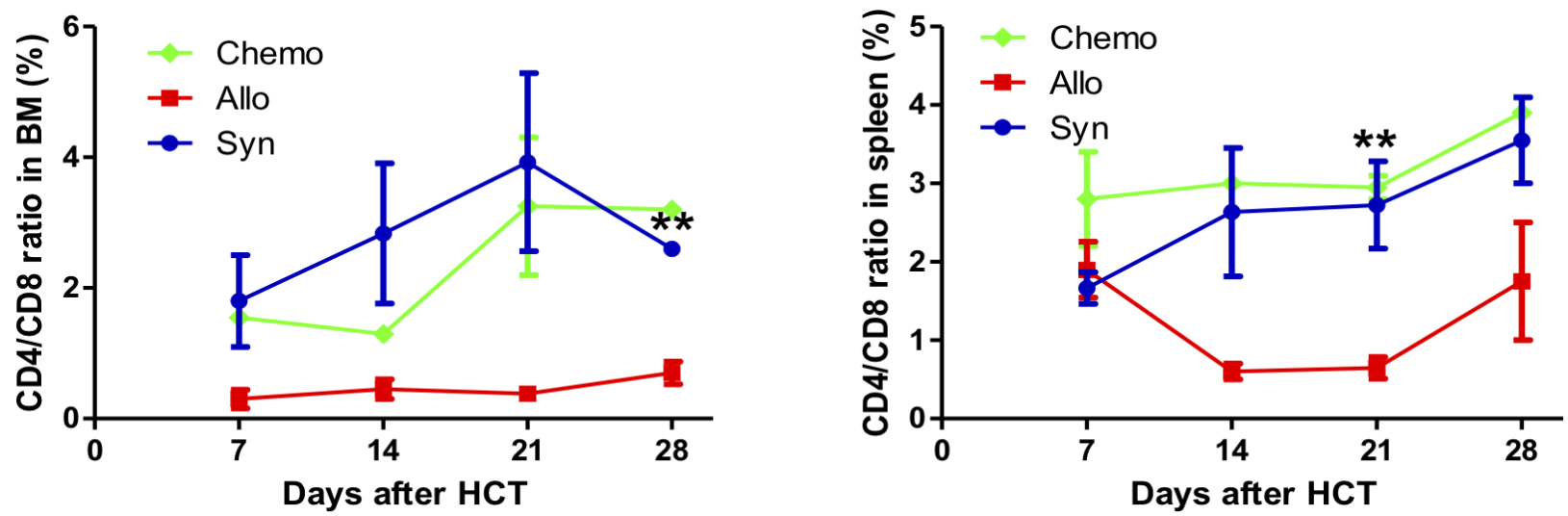


\section{Figure 5}

a

Skin

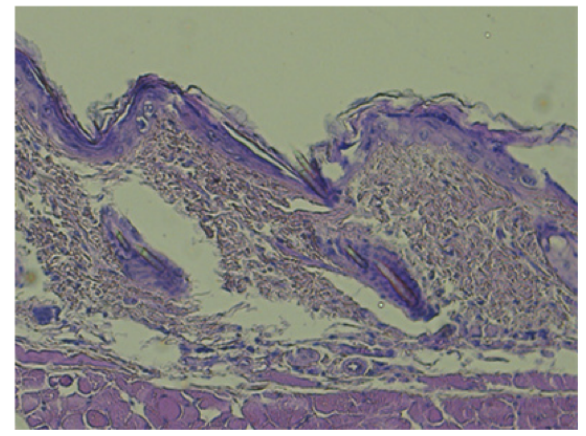

Colon

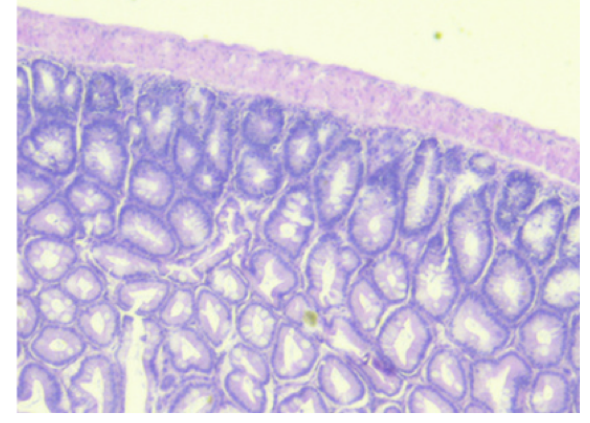

b

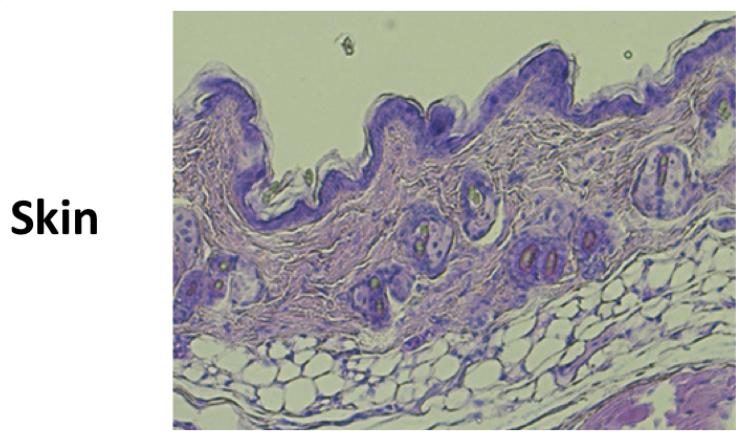

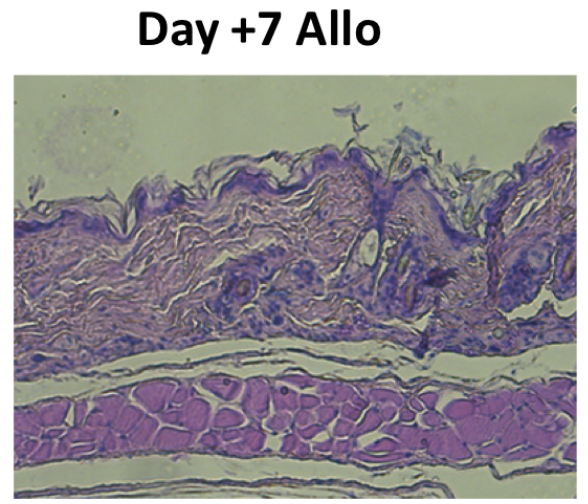

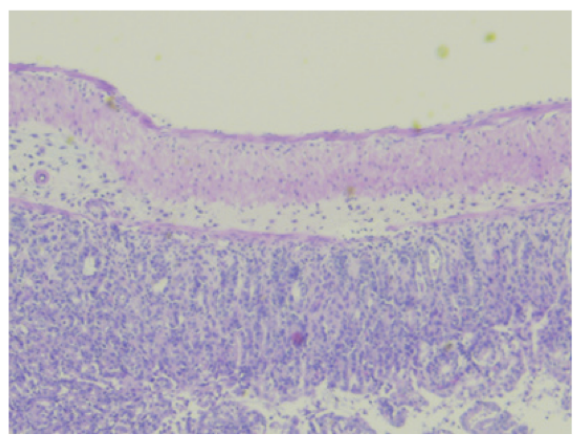

Day +21 Allo
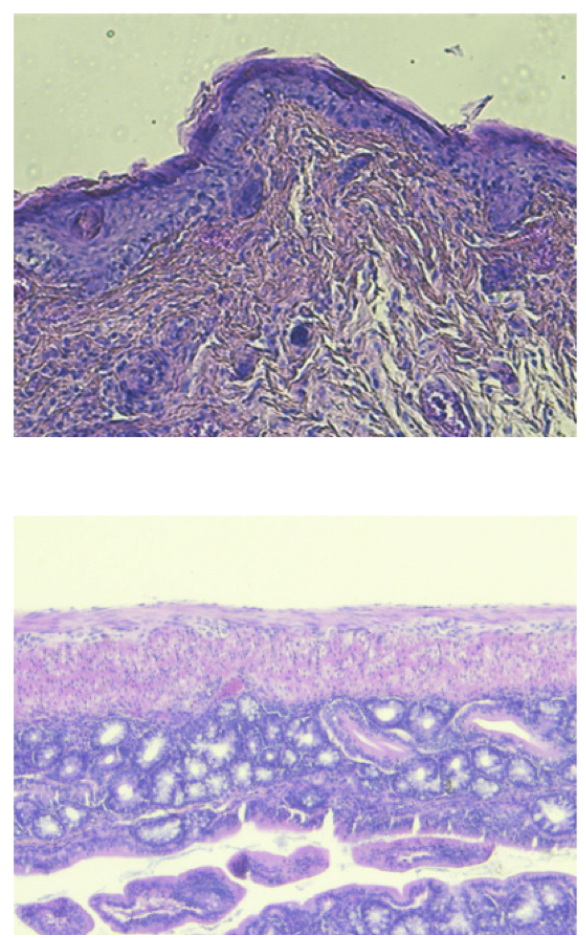

Day +7 Chemo
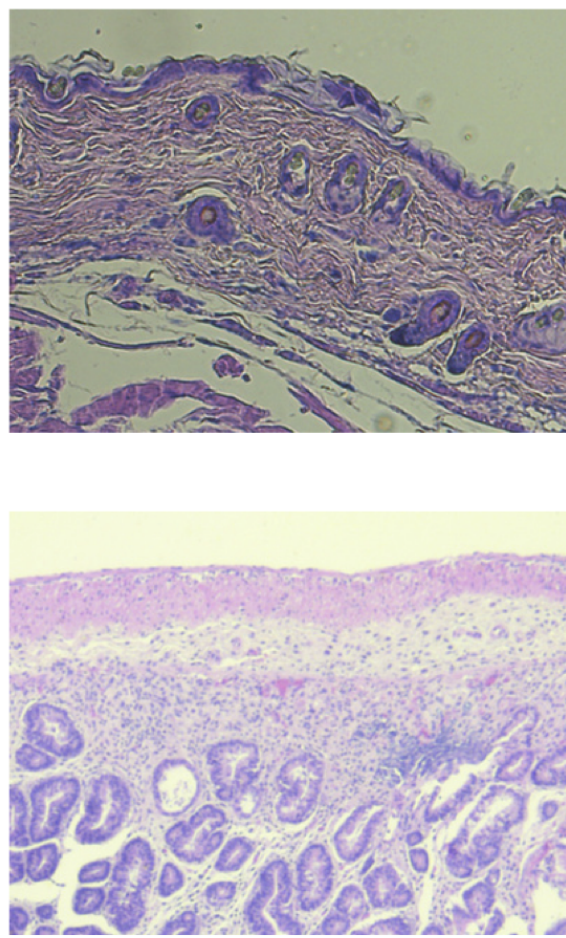

Day +21 Chemo

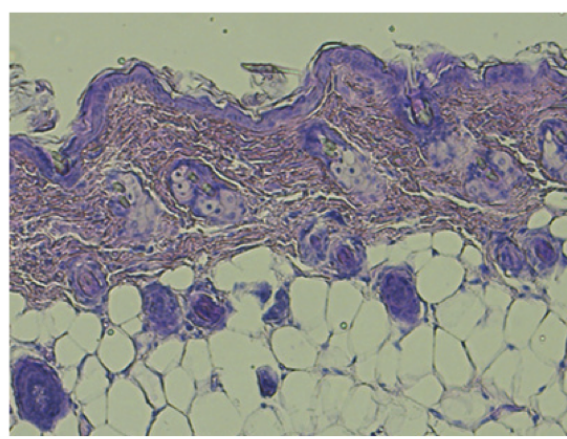

Colon

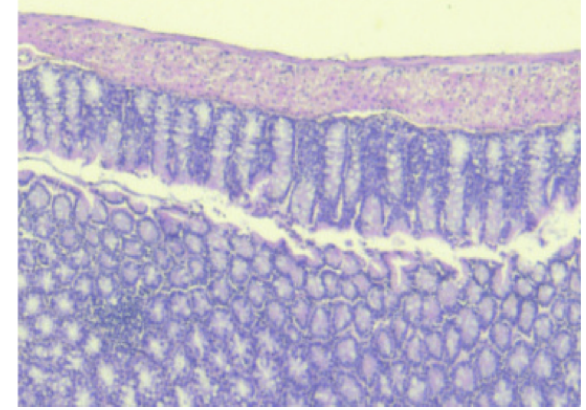

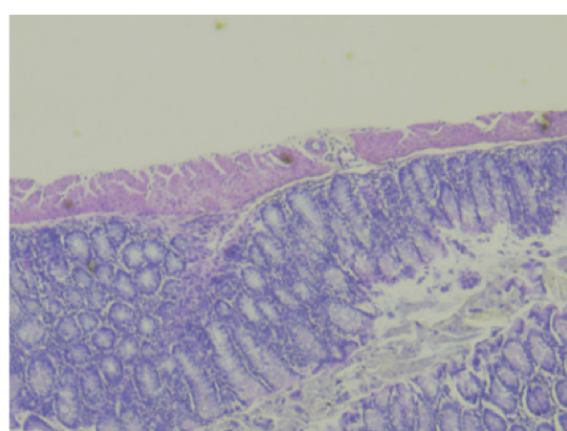


Figure 6

a
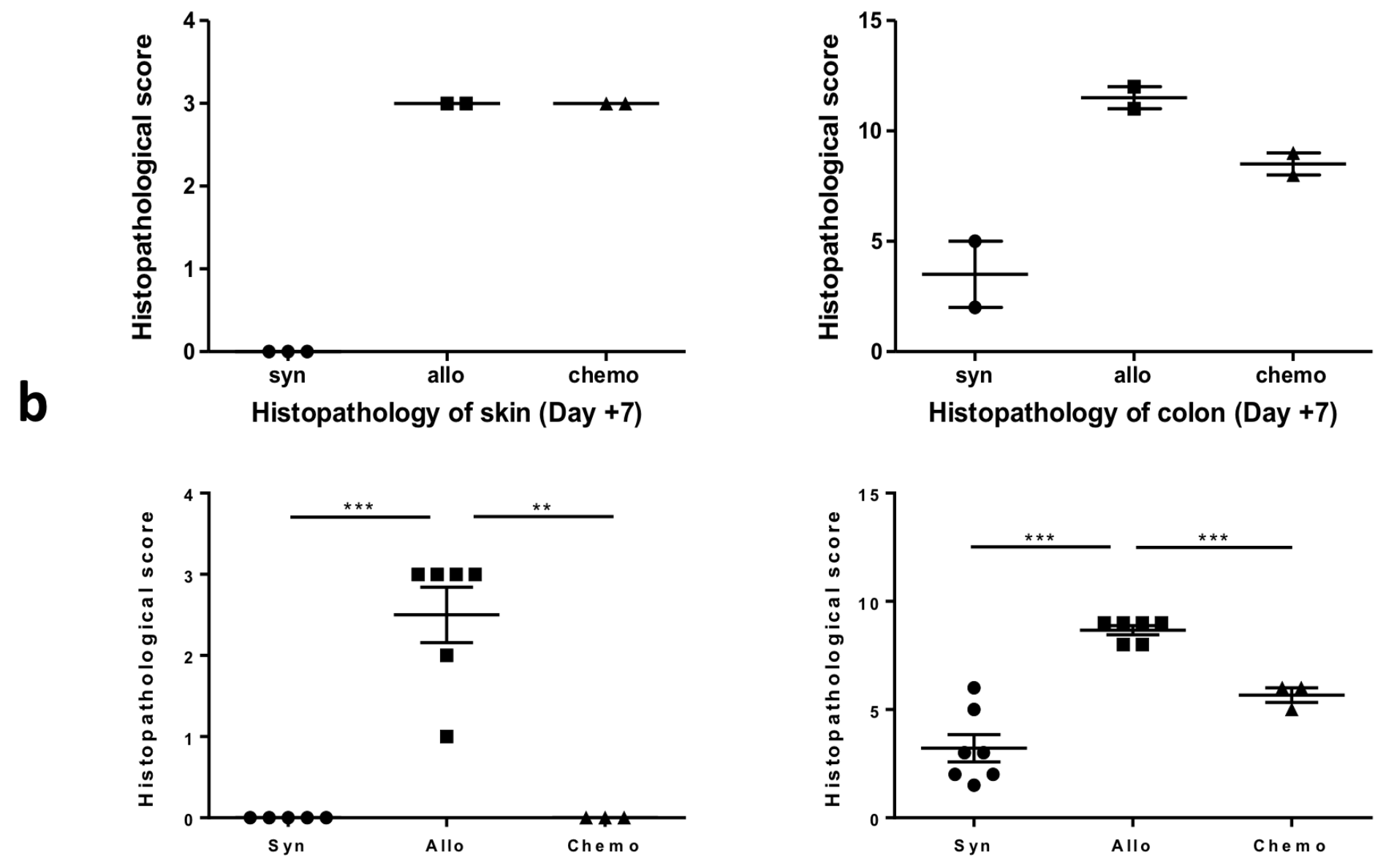

$H$ is to pathology of skin (Day +21) $H$ is topathology of colon (Day +21) 